\title{
El suicidio
}

y otros comportamientos

autodestructivos en jóvenes universitarios

de Colombia y Puerto Rico:

acciones, interacciones

y significaciones

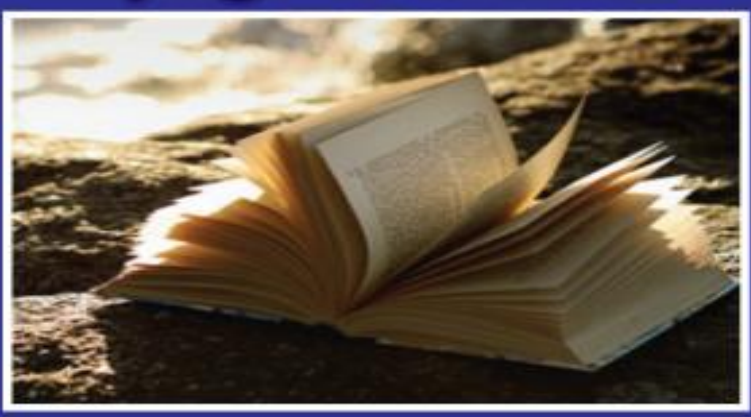

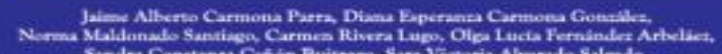

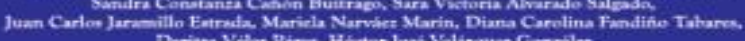

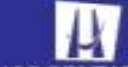

MANIZALES

CAPÍTULO 7. Suicidio y otros comportamientos autodestructivos en jóvenes universitarios de Colombia y Puerto Rico: análisis comparativo.

\section{Modelo de citación}

Carmona Parra, J. A., Cañón Buitrago, S. C., Maldonado Santiago, N., Rivera Lugo, C., Alvarado Salgado, S. V. y Carmona González, D. E. (2017). Suicidio y otros comportamientos autodestructivos en jóvenes universitarios de Colombia y Puerto Rico: análisis comparativo. En J.A., Carmona, et al. (Comp.). El suicidio y otros comportamientos autodestructivos en jóvenes universitarios en Colombia y Puerto Rico: acciones interacciones y significaciones. (pp. 143-158). Manizales:

Universidad de Manizales. 


\title{
7. Suicidio y otros comportamientos autodestructivos en jóvenes universitarios de Colombia y Puerto Rico: análisis comparativo
}

\author{
Jaime Alberto Carmona Parra \\ Sandra Constanza Cañón Buitrago \\ Norma Maldonado Santiago \\ Carmen Rivera Lugo \\ Sara Victoria Alvarado Salgado \\ Diana Esperanza Carmona González
}

Los dos capítulos de resultados que se van a comparar en este apartado se construyeron con las narrativas aportadas por los estudiantes que diligenciaron el segundo instrumento. La condición de inclusión, en este caso, consistió en que los jóvenes hubieran trabajado el primer instrumento y que en éste afirmaran haber realizado uno o más intentos de suicidio en el último año, o bien haber presentado ideaciones suicidas, o haber tenido conocimiento cercano del suicidio o el intento de suicidio de un estudiante universitario.

La decisión metodológica de incluir en este análisis las ideaciones, los intentos y los suicidios consumados, deriva de que el interés de esta investigación no apunta específicamente a uno de estos tres procesos en particular, sino a los comportamientos autodestructivos, que son concebidos como procesos que inician desde la ideación, pasan por comportamientos de negligencia en el autocuidado y temeridad, y culminan 
con los intentos y los suicidios consumados. La decisión de incluir las cuatro poblaciones responde a la intención de construir una narrativa sobre el fenómeno no limitada a la visión de un solo actor social, sino que establezca un diálogo a cuatro voces de actores sociales que comparten en común el rasgo de ser estudiantes universitarios y que han vivido de cerca el fenómeno, pero desde posiciones diferentes respecto al mismo. Para efectos del análisis, cuando aparecen diferencias importantes, se presentará la información diferenciada por comportamiento, por tipo de población, o por ambas variables, y en los casos que las narrativas tiendan a coincidir se presentarán de manera global.

\subsection{Proporción por sexo, método y día de la semana}

De los estudiantes que realizaron un intento de suicidio en el último año, en los dos países, la mayoría fueron mujeres, lo que concuerda con las tendencias a nivel internacional (OMS, 2014) registradas por los equipos de investigación de Colombia y Puerto Rico. La OMS estima que la proporción de intentos a nivel mundial es de tres mujeres por cada hombre. Es pertinente aclarar, sin embargo, que la tasa de suicidio es más alta para hombres que para mujeres (OMS, 2014; INEGI, 2015).

En las respuestas del estudiantado colombiano el método predominante fue la intoxicación seguido por la automutilación. En Puerto Rico, la automutilación y la intoxicación mostraron una proporción similar.

En cuanto al día de la semana, tanto en Colombia como en Puerto Rico, las ideaciones son más frecuentes entre viernes y domingo. Sin embargo, en ambos países, la mayoría de los intentos de suicidio y los suicidios en el último año ocurrieron entre lunes y jueves y la mayoría de los intentos se dieron entre la tarde y la noche, durante el período académico. La mayoría de los participantes reportó que los intentos de suicidio y los suicidios ocurrieron en la propia casa de los jóvenes.

Hay diferencias entre los métodos de quienes se suicidan, quienes lo intentan y quienes tienen ideaciones. En los primeros se destaca la frecuencia del ahorcamiento, en el segundo predominan las automutilaciones y la intoxicación, y para el tercero permanece la intoxicación y el chocar el auto.

Se encuentran, asimismo, diferencias con relación al cuándo. Para las ideaciones sobresale el fin de semana, lo cual contrasta con los días de intentos y suicidios ya señalados. Por otra parte, las ideaciones se dan con mayor frecuencia en el transcurso del semestre, coincidiendo con los intentos y los consumados. 


\subsection{Acciones, interacciones y significaciones relacionadas con los comportamientos autodestructivos de los estudiantes}

\subsubsection{Acciones}

Con respecto a la temporalidad de las acciones, acontecimientos o hechos que los estudiantes sitúan como relevantes, en relación con sus comportamientos autodestructivos, en ambos países sobresalen los acontecimientos del presente.

Al considerar acontecimientos del pasado predominan las referencias a decepciones radicales con adultos significativos, deprivaciones afectivas, violencias sexuales y relaciones conflictivas con parientes. Respecto a los acontecimientos presentes, se encuentran las situaciones de tensión y conflicto con otros significativos, parientes en la mayoría de los casos. Más de la mitad de las narrativas referidas a eventos o situaciones de la vida actual, en ambos países, se relacionan con tensiones vinculares. Es importante destacar la diferencia radical entre el peso relativo de los conflictos en las relaciones vinculares y otras condiciones psicosociales como pérdidas económicas, enfermedades, fracasos académicos o laborales, incluso fallecimientos de otros significativos. Llama la atención que un acto tan radical como el atentar contra la propia vida se describa con mayor frecuencia utilizando narrativas como: "mi mamá es fuerte de carácter, me comparaba con mi hermana mayor, tenía prejuicios conmigo por mi forma de vestir" o "el hecho de no ver frutos en cuanto a los estudios" (Puerto Rico) o "los papás son separados, y ella vive sola con la mamá, pero lastimosamente la mamá casi no mantiene con ella" (Colombia), que con otras narrativas relacionadas con problemas objetivos como: "la cantidad de problemas que tengo de tipo económico". Esto nos sugiere que, según los propios jóvenes, entre los acontecimientos que empujan al suicidio, las tensiones interpersonales actuales con sus familias, parejas y amistades juegan un papel fundamental, mucho más que los eventos de su infancia y que otros eventos objetivos del presente

\subsubsection{Las interacciones}

Es pertinente señalar la alta coincidencia en los resultados en ambos países en cuanto al peso relativo que tienen las interacciones familiares sobre los demás vínculos. El segundo lugar, en Colombia, lo ocupan las interacciones de pareja, y en Puerto Rico, 
los vínculos con compañeros y amistades. Quizás lo más significativo a destacar, respecto a las interacciones familiares, consiste en que más de la mitad de las respuestas en ambos países no se refieren a un vínculo en particular sino a la familia como conjunto, o a dinámicas colectivas que involucran a dos o más integrantes de la familia y en las que suele estar presente uno de los padres o ambos. Por ejemplo en Colombia: "mi mamá, mis dos hermanas"; "pues mis padres y un hermano ya que ellos son los causantes". Asimismo en Puerto Rico: “en ese momento en que se nubló mi mente pensé que podía salir de todo de una forma rápida y así lograría la felicidad de ellos [mi familia]." Sobre las narrativas referidas a un individuo en particular, predominan las dirigidas a la madre y al padre, con más frecuencia a la primera.

\subsubsection{Las significaciones}

A continuación abordamos los resultados obtenidos a partir de las preguntas orientadas a establecer las significaciones (ideas e ideales) asociadas con los comportamientos autodestructivos (ideaciones, intentos y suicidios). Se señalan los campos de significación más importantes que aparecieron, empezando por el de mayor frecuencia. La primera significación: “abandono, soledad y desamor” arrojó una amplia concordancia en las narrativas de los estudiantes de ambos países, y de igual manera la segunda significación: "alivio, liberación o solución radical”.

\subsubsection{Abandono, soledad y desamor}

Se trata de una significación con una fuerte carga afectiva donde se acentúa la idea de una carencia de vínculos o de una pobreza afectiva de los mismos, lo cual es vivido por los jóvenes como el origen de una profunda tristeza que, influenciados por el lenguaje en boga, llaman “depresión”. Veamos narrativas típicas de los dos países: “que nadie me quería", "porque no le importaba a nadie" (Colombia); "se sentía sola y abandonada, tenía poca interacción con sus amistades", "el no sentirme querida por las personas que me rodean" (Puerto Rico).

\subsubsection{Alivio, liberación o solución radical}

En esta significación agrupamos las narrativas de los jóvenes que se ven a sí mismos como acorralados por su entorno o sus circunstancias. Sobresalen las referencias a situaciones que conllevan elevados niveles de estrés. A diferencia de la tipología anterior, en la que se destaca la desidia o indiferencia de los otros, en este caso los otros aparecen como una hiperpresencia que acosa y agobia. Veamos narrativas 
típicas: "estas ideas consisten en acabar con mi vida, en desaparecer de este mundo para no sufrir más y no ver más sufrimiento”, "debo dejar de sufrir, ya estoy cansada, necesito encontrarle paz a mi vida” (Colombia); "que todos los problemas que tenía en ese momento no tenían solución [... ] lo veía como una alternativa de liberar todo”, “en verdad que estoy que no aguanto más, siento demasiada presión y siento que me voy a colgar en mis clases" (Puerto Rico).

\subsubsection{Respuesta frente a una decepción radical}

En casos de una decepción radical, la idea del comportamiento autodestructivo aparece con una carga de agresión hacia los otros, como una especie de lección que les quiere dar el suicida, privándolos de su existencia. Es notoria la diferencia con las descripciones anteriores en las que el participante aparece a merced de los otros que lo abandonan y lo desprecian, o lo presionan y agobian. En la respuesta frente a una decepción radical el joven carga su acto con un fuerte sentido, intencional, de afectar a los otros: "Consisten en pensar de qué manera puede uno suicidarse y cómo las demás personas lo pueden llorar o extrañar" (Colombia); “en realidad, cuando pensaba en mis padres sentía más curiosidad de cómo ellos reaccionarían a mi muerte” (Puerto Rico).

\subsubsection{Pensamientos de autodevaluación}

El comportamiento autodestructivo puede estar ligado a una representación particularmente negativa de la misma persona. Estas ideas negativas suelen ser interiorizaciones de los juicios que otros significativos han emitido sobre los jóvenes, en el pasado o el presente. Se ubican en esta categoría porque en el momento en que son enunciadas por los jóvenes ya se han internalizado, y la acción autodestructiva se asume como una consecuencia de esa idea de sí mismos: "no soy nadie; para qué vivo"; "es mejor no seguir viviendo ya que uno es un estorbo para esta vida y la familia"; "me sentía torpe" (Colombia); "nadie me quiere, todo siempre es mi culpa [... ], si acabo con mi vida le hago un favor a la sociedad"; "desde pequeña fui el "error" de mi madre y me lo echaba en cara todo el tiempo..." (Puerto Rico).

\subsubsection{La idea de la falta de sentido de la vida}

La afirmación del sin sentido de la vida es en sí misma una significación: "porque no tenía un propósito en mi vida”; “consisten en que no encuentro un sentido de vida a pesar de que tengo la oportunidad de estudiar y estar en la universidad" (Colombia). "No creo que la vida tenga un sentido propio, es solo una forma de respirar mecánica" (Puerto Rico). A manera de ilustración, las siguientes narrativas aluden indirectamente al 
sin sentido: "perdí la fe, espíritu, en ser feliz" (Colombia), “...no quería responsabilidades y no le encontraba motivo a la vida" (Puerto Rico).

\subsubsection{La ausencia de un significado asociado a la idea de quitarse la vida}

Una sexta tipología abarca narrativas en las que no se identifica significado particular alguno que acompañe el comportamiento autodestructivo. Aparece solamente la idea de morir o quitarse la vida y el temor al acontecimiento. Por ejemplo: “... imágenes aterradoras de cómo podría morir, ser pisada por un automóvil, que el bus en el que voy se voltee, colgarme desde un palo que hay en mi pieza, pero después, sólo rezo y pido a Dios que no se vayan a cumplir"; "cómo podría morir, pero me da miedo del dolor, por lo mismo no me atrevo a realizarlo y no sé qué pasaría, me causa mucha inquietud" (Colombia). "Yo no puedo hacer ésto..., pero es que no quiero seguir viviendo... una lucha interna”; “en realidad me abundó el miedo a dejar de existir en la tierra, no de vivir, porque realmente no quería vivir”. (Puerto Rico).

En primer lugar, hay que resaltar las profundas coincidencias que encontramos en las narrativas de los estudiantes de los dos países, lo cual puede explicarse, entre otras, por las coincidencias culturales en lengua, religión, historia y economía. Adicionalmente ambos países están ubicados en la región tropical de América. Estas coincidencias muestran la aplicabilidad de los resultados de esta investigación para los dos países. También sugieren auscultar las posibilidades de seguir explorando la presencia e incidencia de la dimensión cultural en los comportamientos autodestructivos de los jóvenes universitarios en otros países latinoamericanos.

El análisis de las narrativas también nos permite constatar la íntima relación que existe entre las acciones, las interacciones y las significaciones en el estudio de los comportamientos autodestructivos. Si bien cada una de estas categorías tiene su propia especificidad, todas contribuyen a iluminar un costado del fenómeno. Asimismo, se evidencian importantes zonas de intersección entre ellas.

En el análisis presentado en torno a la relación de los comportamientos autodestructivos con acontecimientos, eventos o hechos, se sitúan algunos, como las violencias físicas, sexuales y simbólicas. Dentro de estas últimas, se incluyen las prácticas de exclusión, acoso y segregación que se dan dentro de los diferentes contextos de interacción. También se consideraron acontecimientos asociados con decepciones 
respecto a otros o a sí mismo. Otro grupo de acontecimientos refiere a pérdidas de seres queridos por fallecimiento, reveses y penurias debidas a la situación socioeconómica u otro tipo de tragedias. Es importante destacar la diferencia radical del peso relativo de los conflictos intersubjetivos en los vínculos con los otros significativos, sobre otros acontecimientos en los que la dimensión intersubjetiva, aunque ciertamente presente, no necesariamente se muestra con tanta claridad, como las pérdidas económicas, las enfermedades, los fracasos académicos o laborales, incluso las pérdidas de seres queridos.

Las violencias que mencionan los jóvenes no provienen de anónimos, ni de cualquier clase de actor social, sino precisamente de los más íntimos, con lo cual se entra en la zona de intersección de las acciones y los vínculos; por tanto, lo que define un vínculo y lo diferencia de otro es el proceso de significación, y con ello se entra en la zona de intersección de los vínculos y las significaciones. Otro tanto se tendría que decir del abordaje realizado de los vínculos: no es gratuito que a nivel individual sean la madre y el padre, en su orden, los vínculos que aparecen asociados con más fuerza a las narrativas sobre comportamientos autodestructivos, y que sea la familia como espacio vincular colectivo el que aparezca como el más importante.

\subsubsection{El papel de la universidad en los comportamientos autodestructivos de los estudiantes}

Al final de la sección orientada a la exploración de las acciones, interacciones y significaciones relacionadas con los comportamientos autodestructivos, se les hizo a los estudiantes la siguiente pregunta ¿Hubo alguna circunstancia relacionada de manera directa o indirecta con la vida académica, que no haya mencionado en las respuestas anteriores? A continuación, se analizan los resultados de las narrativas aportadas a partir de esta pregunta.

La vida universitaria impone a los jóvenes estudiantes una serie de cambios que demandan múltiples y variados ajustes y adaptaciones para enfrentar sus estudios exitosamente. Estudiar en la universidad supone, para muchos estudiantes, enfrentar un sinnúmero de retos y desafíos, como vivir separados de la familia por primera vez, experimentar sentimientos de soledad o aislamiento social y sentir nostalgia por amistades de la secundaria (en Colombia) o la escuela superior (en Puerto Rico). El comienzo de los estudios universitarios, con frecuencia acarrea el tener que asumir mayor autonomía e independencia para manejar asuntos relacionados con sus finanzas,

vivienda, transporte y salud, entre otros. Todo ello, sumado a los retos que suponen 
nuevos estilos de enseñanza/aprendizaje y demandas académicas que convierten la experiencia universitaria inicial en un reto. Además, conviene considerar la posibilidad de que problemas de progreso académico con frecuencia pudieran ocultar comportamientos potencialmente suicidas. Es por tanto particularmente importante procurar información confiable de factores de riesgo y factores de protección en este grupo.

Los resultados demuestran que muy pocos estudiantes, que realizaron algún intento de suicidio en el último año, relacionaron directamente la acción autodestructiva con su vida académica. Por otra parte, los estudiantes que presentaron ideaciones suicidas señalaron alguna relación, y de igual manera los estudiantes que tuvieron conocimiento de un suicidio consumado.

El análisis de contenido de las narrativas arroja que, para la mayoría de los estudiantes, el papel de la universidad en los comportamientos auto- destructivos es circunstancial: "simplemente que la chica estudiaba con él, es la única relación con la academia" (Colombia). Otros ejemplos muestran que las presiones familiares afectan el papel de la universidad y tienen una influencia importante en la construcción de la acción autodestructiva, bien sea por las presiones en la elección: "insatisfacción con su carrera, no era precisamente lo que quería estudiar"; o por su situación económica: “a él le estaba yendo súper mal en las materias y él sabía que pagar su carrera era un esfuerzo grandísimo para su familia, por lo injustamente costosa que es la educación superior, por eso él se sentía muy mal”. El desempeño académico es otra instancia en las que se agudiza el problema: "la falta de motivación hacía que no realizara bien sus deberes y reprobara las materias, este último factor aumentaba su estrés y disminuía su entusiasmo" (Puerto Rico).

Ahora bien, literatura revisada (Wilcox, Arria, Caldeira, Vincent, Pinchevsky, \& O'Grady, 2010; Jiménez, 2009; Drum, D, Brownson, Denmark, \& Smith, 2009; Bentancurt, 2006; Appelbaum, 2006) evidencia que la presencia de condicionantes como competitividad académica, presión de grupo, dificultades académicas y económicas, relaciones despersonalizadas con docentes y administración, son ciertamente factores específicos propios de dinámicas institucionales, que contribuyen al incremento del fenómeno del suicidio en el escenario universitario.

Finalmente aparecen referencias a situaciones en las que los estudiantes y combinan sus estudios con la actividad laboral, situación que, tanto en Puerto Rico como en Colombia, tiende a ser cada vez más generalizada: "en las universidades los docentes sobrecargan a los estudiantes de trabajos, sin considerar que la gran mayoría 
de estudiantes trabajan y estudian al tiempo, sin contar los que son padres de familia" (Colombia). Este puede ser un Ilamado para que desde las universidades se oriente adecuadamente al estudiantado para que tome el número de asignaturas que pueda cursar de manera óptima, sin exponerse a situaciones de estrés que los excedan. Se trata de escuchar para aprovechar las posibilidades preventivas que existen de manera potencial en cada uno de los actores y de las interacciones del escenario de la educación superior, y convertir a las universidades en promotoras de dinámicas que fortalezcan las competencias para la vida y contribuyan a la prevención de comportamientos autodestructivos en el estudiantado universitario.

\subsection{Acciones, interacciones y significaciones protectoras ante los comportamientos autodestructivos de los estudiantes}

Así como se han identificado múltiples situaciones que representan un factor de riesgo para las conductas autodestructivas en los jóvenes, estas mismas situaciones pueden ser, para otra persona un factor protector. Como lo plantea Carmona (2012): es necesario tener en cuenta "la autodeterminación del sujeto... una capacidad de agencia variable de acuerdo con el contexto de interacción, el rol que el actor social desempeña en él y la coyuntura específica en la que se lleva a cabo la acción” (p. 320). Además,

Todos nuestros vínculos están organizados por juegos de significados y que no existen acciones humanas que puedan abstraerse del vínculo y el contexto socio-simbólico en el que se producen. Una caricia o una agresión no son asuntos que ocurren en el vacío, se dan en el contexto de una interacción, con un padre, un par, un superior, un extraño...y esa interacción anudada a las circunstancias que la rodean son los elementos que le dan su significado último. (Carmona y Cañón, 2016, p. 113)

Se exponen a continuación acciones, interacciones y significaciones que los estudiantes, tanto de Colombia como de Puerto Rico, consideraron como factores protectores ante los comportamientos autodestructivos. 


\subsubsection{Acciones}

La pregunta formulada a los estudiantes que habían presentado intentos de suicidio o ideaciones suicidas en el último año fue la siguiente: ¿Qué acciones o hechos concretos realizados por otras personas o por usted mismo, ocurridos en la época de su intento (o sus ideaciones) y después, considera usted que fueron fundamentales para evitar que usted se suicidara o para ayudarle a luchar por seguir viviendo?

Advertimos que las respuestas de los estudiantes no siempre se ajustan a la consigna de referir "acciones" o "hechos". Con frecuencia sus respuestas remiten a un vínculo o a una significación. En cierto sentido, confirman lo señalado con anterioridad, cuando se advertía que, en el campo de la experiencia humana no hay acciones en el vacío, toda acción se da en el marco de una interacción y, a su vez, las interacciones están organizadas en sistemas de significaciones. De ahí que sea correcto afirmar que en la experiencia humana una acción, y de una manera especial una acción autodestructiva, solamente puede comprenderse en función del contexto de interacciones y significaciones en el que se desarrolla.

Siguiendo las tendencias de las narrativas de los estudiantes, se organizaron las acciones mencionadas en las respuestas a esta pregunta por su ubicación en un campo de vínculos determinado. En ambos países son mayoritarias las respuestas en las que el joven se atribuye a sí mismo la autoría de las acciones que le ayudaron a superar sus comportamientos autodestructivos y afirmarse en el deseo de vivir. Es llamativo que en este tópico, en el que el protagonismo de la acción protectora recae sobre el mismo actor social que realizó la acción autodestructiva, la proporción de las narrativas de Puerto Rico duplica la de Colombia: "rezar"; "ir a misa"; "hacer catarsis"; "llorar"; "tener sexo"; "iniciar terapias psicológicas"; "tener empleo (Colombia). En Puerto Rico: "Clases de zumba"; "ir a servicios religiosos en la iglesia"; "acciones benéficas y trabajo comunitario"; "visitar a grupos de jóvenes"; "tomar cursos de Psicología"; "participar de talleres de arte y desarrollo profesional"; "cambio de institución universitaria y de carrera profesional".

La importancia de estas citas reside en que provienen de los mismos estudiantes que han estado en riesgo de suicidio, en torno a las acciones que les han servido para afirmarse en el mundo. Aparte de que algunas puedan resultarnos anodinas o banales, merece detenerse a pensar en ellas como orientaciones que ellos mismos aportan para construir estrategias de prevención. 
El segundo ámbito en el que se sitúan las respuestas de los estudiantes a la pregunta por las acciones protectoras difiere de un país al otro. En Colombia es la familia: "ser madre y estar con su hijo"; “cuidado permanente de mamá y hermana"; "sentir y darse cuenta del cariño de los padres hacia sí mismo"; "el valor recibido en la familia de origen y familia extensa”. En Puerto Rico es el ámbito de relaciones de amistad y de pareja: "tener invitaciones a salir"; "recibir llamadas y demostraciones de afecto".

El peso significativamente mayor de las acciones individuales y la marginalidad de las acciones referidas al ámbito familiar, en las narrativas de los estudiantes de Puerto Rico, con respecto a la juventud colombiana (donde la proporción de las acciones individuales es la mitad, y las acciones correspondientes al ámbito familiar son casi el triple) quizás pueda explicarse por la influencia del estilo de vida norteamericano, que ciertamente es mucho más fuerte en la Isla que en Colombia. En este punto hay un elemento diferenciador que puede ser útil a los servicios de bienestar universitario de ambos países para trazar estrategias diversas.

\subsubsection{Interacciones}

En este punto los resultados de los instrumentos ofrecen un panorama que difiere un poco de las narrativas correspondientes a la pregunta anterior. No obstante, se presentan los resultados tal como aparecen en las respuestas de los estudiantes a la siguiente pregunta: ¿En la época anterior, en la época del intento o posteriormente, hubo vínculos (familiares, de amistad, amorosos, académicos, compañeros de trabajo y otros) que usted puede reconocer que le ayudaron a seguir viviendo? Mencione los más significativos en ese momento y la manera concreta en que influyeron.

En Colombia, los vínculos de pareja y amistades tuvieron el porcentaje mayor de respuestas, mientras que las respuestas relativas a la familia fueron menores. En Puerto Rico, la familia obtuvo un porcentaje mayor de las respuestas que la pareja y las amistades, aunque la diferencia no es considerable. En ambos países hay dos coincidencias, la primera es que, en el ámbito familiar, la mención de la familia como conjunto supera ampliamente la referencia a uno o algunos de los integrantes; la segunda se refiere al campo de la amistad y las relaciones de pareja, en que también las referencias a las relaciones de amistad superan ampliamente las menciones de las relaciones de pareja. Lo anterior permite resaltar el valor de las relaciones afectivas de amistad para los jóvenes. De estos elementos que aportan los jóvenes se pueden derivar valiosas herramientas para la prevención. 


\subsubsection{Significaciones}

La pregunta orientadora para este tópico fue la siguiente: "Mencione si hubo ideas contrarias al suicidio que le ayudaron a seguir viviendo o a evitar que las ideaciones o el intento llegara a las últimas consecuencias (la época anterior, en la época del intento o posteriormente). Si las recuerda le pedimos que las transcriba tan literalmente como pueda".

Observamos en los estudiantes de Puerto Rico que los pensamientos de la posición mayoritaria corresponden al tópico del pensamiento autocrítico, seguidos por las ideas relacionadas con la familia. Para el caso colombiano, los dos primeros lugares los ocupan los tópicos relacionados con la familia y los amigos, compañeros y parejas, con un porcentaje igual.

Quizás lo más valioso de este apartado no sean las diferencias sino las coincidencias, más que en los porcentajes de varios de los tópicos, en el contenido de las narrativas. Tales coincidencias posibilitan admitir que los jóvenes estudiantes universitarios están brindando orientaciones sobre ámbitos de significación a los es posible apelar para la construcción de estrategias de prevención ante las acciones autodestructivas.

El tópico que en capítulos anteriores se denominó "pensamiento autocrítico", se refiere a narrativas en las que los jóvenes dan cuenta de su capacidad de tomar distancia de las propias significaciones que los llevaron a poner en riesgo sus vidas. Se trata de una operación mental en la que se establece una especie de discusión interna, en la que aparecen pensamientos alternativos e incluso opuestos a las ideas autodestructivas y logran hacerse valer en contra de éstos. Por ello se emplea la expresión "pensamiento autocrítico", porque implica la posibilidad de ser crítico frente a las propias ideas, lo cual requiere de una disposición subjetiva flexible que soporte la contradicción interna, incluso que contemple el elemento del humor, entendido como la capacidad de reírse de sí mismo. De nuevo se comprueba cómo los supuestos del interaccionismo simbólico dan las coordenadas para abordar la prevención del suicidio en el ámbito universitario.

En los dos países hay narrativas en las que el pensamiento autocrítico logra disminuir la gravedad percibida de la situación: "la idea de que todos los seres humanos tenemos problemas y que no soy la única". En otros casos introduce posibilidades no contempladas: "que puedo hacer cosas buenas y productivas por mí y los que me rodean" (Colombia). También permite hacer un examen de los criterios de 
la acción con relación a la manera de interactuar con los otros: "realmente ¿yo quién era? Dejé de ser quien los demás pretendían que fuera, y ya" (Puerto Rico). El pensamiento autocrítico también puede apelar a la comparación del caso propio con otros similares: "el pensar que muchas personas estarían pasando por situaciones mucho más difíciles, me hacían y hacen darme cuenta del error que cometía al tener esos pensamiento negativos" (Colombia). Orientarse a las consecuencias de las ideaciones y las acciones autodestructivas sobre la propia salud: "uno se deteriora de pensar en morir". $Y$, también, el examen crítico directo de la situación con un elevado sentido práctico: "que tenía que buscar el lado bueno de mis problemas, que quitarme la vida no es una solución a mis problemas” (Puerto Rico).

Las significaciones relacionadas con las "expectativas de futuro" permiten disponer la subjetividad en una perspectiva de lo posible y lo deseable, lo cual ayuda a movilizar el pensamiento hacia otros ámbitos diferentes a las acciones autodestructivas. Pero no se limita una función de "distracción" sino que permite resignificar el proyecto de vida de la persona, lo cual necesariamente afecta también las significaciones articuladas con las acciones autodestructivas. Veamos dos significaciones típicas de cada país: "pensar en mi futuro profesional y en el sueño que quiero cumplir"; "que puedo hacer cosas buenas y productivas por mí y los que me rodean" (Colombia); "pensar en cómo sería mi vida si no me quito la vida, cómo sería si yo supero todo esto"; "pensar que podía superarlo, que todavía podía formarme como persona, tener un mejor futuro" (Puerto Rico).

Las significaciones relacionadas con la familia, tienen diversos matices importantes para efectos de la prevención. No se trata solamente de la apelación a la importancia de la familia a secas, sino explorar tópicos particulares de significación. Una primera significación es la familia como fuente de felicidad: "el nacimiento de mi hijo". Otra significación está relacionada con el sentimiento de responsabilidad por el otro: "sacar a mi hijo adelante" (Colombia). También se encontró el deseo de evitar que los seres queridos sufran: "pensar que mi familia puede sufrir con ese hecho" (Puerto Rico). El proporcionarle una alegría especial a alguno de los progenitores: "realmente me gustaría hacer sentir orgulloso a mi papá" (Colombia). U otra significación que puede estar ligada a la anterior, pero en la que se involucra un fuerte componente narcisista: "demostrarle a mi familia, sobre todo a mi mamá, lo que puedo lograr" (Puerto Rico).

También, en las ideas relacionadas con amistades, compañeros y pareja, como significaciones protectoras de las tendencias autodestructivas, es importante considerar la diversidad de matices semánticos, para poder instrumentarlos 
adecuadamente al servicio de la prevención. En algunas de las significaciones el acento puede estar puesto en el pudor y la vergüenza: “también estando ahí, con el cinturón en el cuello, me dio miedo de que mi roommate entrara y me hiciera pasar una vergüenza. Si me veía así, hubiera sido humillante" (Puerto Rico). En otras, lo que puede movilizar a alguien a luchar por la vida es un cierto sentido del orgullo y del logro personal: "mi única idea después del intento, fue que debía de mantenerme viva para poder demostrar de lo que soy capaz" (Puerto Rico). En otros casos, el deseo de sentirse útil para los otros es el movilizador que se puede instrumentalizar para prevenir los comportamientos autodestructivos: "el propósito de la vida y lo que es desde una nueva perspectiva, que es abstracta, dirigida a ayudar a los demás y ya no más centrada solo en esa persona ya que se siente más fuerte en su personalidad" (Colombia). Como se puede apreciar, las orientaciones que aportan los jóvenes con sus narrativas no son fórmulas aplicables de manera automática en todos los casos, sino una especie de caja de herramientas significantes que se pueden implementar, de acuerdo con la particularidad de los estudiantes que acudan en busca de ayuda.

Con respecto a las ideas relacionadas con la espiritualidad y el sentido de la trascendencia, vale recordar que no solamente pueden cumplir su papel preventivo en estudiantes de diversas creencias religiosas, sino también en quienes sin profesar religión alguna asumen al ser humano como ser trascendente. Desde luego, existen diferentes matices en las significaciones de las narrativas que los estudiantes muestran como eficaces para ayudarles a afirmarse en el mundo y luchar por sus vidas. En algunas res- puestas aparece una dimensión alegre de la experiencia religiosa: "Dios me dio su mano y sonrió" (Colombia). En otras la experiencia aparece vinculada con un enigma y el deseo de desentrañarlo: "saber el propósito que Dios tenía para mí" (Puerto Rico). En otros, es el sentimiento de grandeza que proporciona la idea de estar vinculado a un ser superior: "sólo pude dejar mis ideas suicidas cuando encontré un mayor propósito para vivir" (Colombia). Y aún otros que enfatizan el sentimiento de deuda de gratitud y la culpa asociada a él: "pensé en lo mucho que Jesús sufrió por darnos vida, y yo siendo un malagradecido andaba buscando la muerte. Sentí mucho arrepentimiento por mis actos" (Puerto Rico).

Como pueden ver, esta investigación se orientó a mirar el fenómeno desde el punto de vista de los actores sociales y darles la palabra para que sus propias narrativas orienten en la exploración de la complejidad del fenómeno, tanto del origen de las ideaciones suicidas y la construcción de las acciones autodestructivas, como los factores que, según ellos, les han ayudado a seguir viviendo y a afirmarse en el mundo. 


\subsubsection{El papel de la universidad como factor protector ante las acciones autodestructivas del estudiantado universitario}

Para explorar las potencias protectoras de la universidad ante las ideaciones y deseos autodestructivos, se formuló la siguiente pregunta a quienes habían presentado comportamientos autodestructivos o tuvieron conocimiento cercano de un caso de un estudiante que hubiera intentado suicidarse: “¿De qué maneras directas o indirectas considera usted que la carrera, los vínculos y las actividades que se realizan en la universidad, han contribuido para superar el deseo de suicidarse que tuvo (usted o su compañero, según el caso) en aquel momento y afirmarse en el deseo de seguir viviendo y luchar por la vida?".

Las narrativas de los estudiantes de ambos países coinciden en seña- lar tres aspectos fundamentales de la vida universitaria, que operan como factores protectores ante las tendencias autodestructivas, y que se convierten en poderosas motivaciones para afirmarse en el deseo de vivir.

El primero de ellos tiene que ver con la actividad académica en sí misma y la expectativa futura de llegar a ser un profesional y mejorar su calidad de vida: "mi carrera es mi vida, gracias a ella he aprendido el valor y el sentido de la vida tanto en la actualidad, como para mi futuro" (Colombia); “sí, pienso en que seré una profesional próximamente. Pienso en ese futuro que estoy construyendo hoy con mis estudios. Además de que los estudios son el foco de distracción ante todo problema" (Puerto Rico).

El segundo factor se relaciona con los vínculos académicos y de amistad que se construyen en torno a las tareas propias del proceso de formación. Las universidades, además de ser centros de formación profesional, son espacios en los que los estudiantes construyen redes vinculares de calidad: "la relación con algunas personas, sus palabras y actitudes para conmigo, han servido de gran ayuda para comprender que la vida es valiosa sin importar las circunstancias" (Colombia); "entiendo que de cierta manera [la universidad] me ayudó porque conocí más mundo, vi personas que también pasaban por lo mismo que yo" (Puerto Rico).

El tercero es el apoyo directo que puede recibir un estudiante en situación de riesgo por parte de profesores y de profesionales de bienestar universitario: "la atención psicológica constante ha hecho cambiar mis ideas, junto al deseo de superación" (Colombia); "tuve profesores con los cuales compartí experiencias y

sirvieron de consejeros al escuchar y comprender sin juzgar” (Puerto Rico). 
La universidad es un espacio que posee importantes potencias protectoras para la prevención de comportamientos autodestructivos y afirmación del deseo de vivir de los jóvenes. Queda como reto para los equipos administrativos, académicos y de bienestar universitario de las instituciones, potenciar los factores protectores existentes y disminuir los elementos de riesgo, mediante estrategias de generación de acciones, interacciones y significaciones que contribuyan a la prevención de comportamientos autodestructivos y fortalezcan la afirmación del deseo de vivir de nuestros estudiantes.

\section{Referencias}

Appelbaum, P. S. (2006). Law \& psychiatry: Depressed? Get out!: Dealing with suicidal students on college campuses. Psychiatric Services, 57(7), 914-916.

Bateson, G. (1991). Pasos hacia una ecología de la mente. Carlos Lolhé (Trad.). Madrid: Planeta.

Bentancurt, L. M. (2006). Prevención del suicidio en estudiantes universitarios. Una perspectiva psicosocial del fenómeno del suicidio: Los hijos de la desesperanza. Guanajuato Gobierno del Estado: Observatorio de Ciencia, Tecnología e Innovación. Disponible en: http://octi.guanajuato.gob.mx/octigto/formularios/ ideasConcyteg/archivos/08042006_PREVENCION_SUICIDIO_ESTUDIANTES_UNIVE RS.pdf

Capra, F. (1966/1998). La trama de la vida. Anagrama: Barcelona.

Carmona Parra, J. A. (2012). El suicidio: un enfoque psicosocial. Revista Colombiana de Ciencias Sociales, 3(2), 316-339

Carmona, J. A. y Cañón, S. C. (2016). La prevención del suicidio en el escenario universitario. En: J. A., Carmona (Ed.) Ensayos académicos en torno al suicidio. (pp. 101-124). Cali: Fundación Participar IPS.

Drum, D. J., Brownson, C., Denmark, A. B. \& Smith, S. E. (2009). New data on the nature of suicidal crises in college students: Shifting the Paradigm. Professional Psychology, Research \& Practice, 40(3), 213-222.

Instituto Nacional de Estadística y Geografía. (2015). Estadísticas a propósito del día mundial para la prevención del suicidio. Aguascalientes, México. 
Jiménez, M. (2009). Conducta e ideación suicida en estudiantes universitarios. Griot,1, 5-17.

Maldonado S. N., Rivera Lugo, C., Carmona Parra. J. y Jaramillo Estrada, J.C. (2015). Guía para la Prevención del Suicidio en Jóvenes Universitarios: ¿Qué podemos hacer en casos de suicidio, intentos de suicidio e ideación suicida en nuestros estudiantes? Puerto Rico: Pontificia Universidad Católica de Puerto Rico

Morín, E. (1998). Introducción al pensamiento complejo. Barcelona: Gedisa

Organización Mundial de la Salud (2014). Prevención del Suicidio: Un imperativo Global. Washington, D. C. Disponible en: www.who.int.int/mental_health/media/ media_spanish.pdf.

Ruskey, F. \& Weston, M, (2005). A Survey of Venn Diagrams", What is a Venn Diagram? The Electronic Journal of Combinatorics. Recuperado de: http://www.combinatorics.org/files/ Surveys/ds5/VennEJC.html

Wilcox, H. C., Arria, A. M., Caldeira, K. M., Vincent, K. B., Pinchevsky, G. M. \& O'Grady, K. E. (2010). Prevalence and predictors of persistent suicide ideation, plans and attempts during college. J. Affect Disord., 127(1-3), 287-94. doi: 10.1016/j.jad.2010.04.017 